\title{
Future Perspectives on Endoscopic Ultrasonography-Guided Therapy for Pancreatic Neoplasm
}

\author{
Woo Hyun Paik ${ }^{1}$, Sang Hyub Lee ${ }^{1}$ and Sunguk Jang ${ }^{2}$ \\ ${ }^{1}$ Department of Internal Medicine, Seoul National University Hospital, Seoul National University College of Medicine, Seoul, Korea, \\ ${ }^{2}$ Department of Gastroenterology and Hepatology, Cleveland Clinic, Cleveland, OH, USA
}

Endoscopic ultrasonography (EUS)-guided therapy with ethanol injection or catheter-based radiofrequency ablation for pancreatic neoplasm has been conducted as a potential alternate treatment modality for patients who are not eligible for surgery. On the basis of the limited number of studies available, EUS-guided ablation therapy with the aforementioned methods for small pancreatic neoplasms has demonstrated promising technical feasibility and safety profiles. To be considered as a legitimate alternative option to surgery, however, EUS-guided ablation therapy must provide a long-term efficacy profile along with the consensus among experts regarding its treatment parameter. This review focuses on the clinical issues and future perspectives of EUS-guided therapy for pancreatic neoplasm. Clin Endosc 2018;51:229-234

Key Words: EUS; Pancreatic neoplasms; Treatment outcome; Contraindications; Ethanol

\section{INTRODUCTION}

The incidence and prevalence of pancreatic neoplasm are increasing rapidly with the development and diffusion of various cross-sectional imaging modalities. ${ }^{1-3}$ As the biological behavior of pancreatic neoplasms is highly heterogeneous, constructing a standardized protocol for its treatment and follow-up remains a significant challenge. Although surgical resection is the definitive treatment for pancreatic neoplasms with malignant transformation potential, it carries relatively high risks of perioperative morbidity and mortality. ${ }^{4}$ Over the last decade, endoscopic ultrasonography (EUS)-guided ablation of pancreatic neoplasms has been performed as an experimental treatment modality for patients who were either

Received: April 9, 2018 Revised: April 26, 2018

Accepted: April 27, 2018

Correspondence: Sunguk Jang

Department of Gastroenterology and Hepatology, Digestive Disease Institute, Cleveland Clinic, 9500 Euclid Ave, Q3-1, Cleveland, OH 44195, USA

Tel: +1-216-636-5440, Fax: +1-216-444-6284, E-mail: jangs@ccf.org ORCID: https://orcid.org/0000-0001-9837-0322

(c) This is an Open Access article distributed under the terms of the Creative Commons Attribution Non-Commercial License (http://creativecommons.org/ licenses/by-nc/3.0) which permits unrestricted non-commercial use, distribution, and reproduction in any medium, provided the original work is properly cited. poor surgical candidates or reluctant to undergo surgery. ${ }^{5,6}$ A recent retrospective study that compared patients who received EUS-guided ethanol injection for the ablation of cystic neoplasms with those who did not revealed that the patients treated with EUS-guided ablation with ethanol injection maintained their quality of life by avoiding pancreatic surgery ${ }^{7}$

Although EUS-guided ablation therapy for pancreatic neoplasms has been investigated for over 15 years, a clear consensus on its efficacy remains elusive; hence, it has yet to become a part of the standard of care. ${ }^{8}$ Studies on EUS-guided ablation therapy have reported a wide range of efficacies, with reported resolution rates of pancreatic cystic neoplasm ranging from $9 \%$ to $79 \%{ }^{9-14}$ Data on EUS-guided ablation therapy for pancreatic solid neoplasm are even scarcer. To date, the reported rates of complete resolution of pancreatic solid tumors range from $62 \%$ to $100 \%{ }^{15-18}$ Given the substantial heterogeneity of the reported efficacy of EUS-guided ablation therapy, each step of the procedure should be evaluated to optimize efficacy. Furthermore, the procedure-related safety issues, including the occurrence of pancreatitis, vascular injury, and infection, and the risks of tumor seeding, recurrence or metastasis need to be studied further. ${ }^{19}$ The clinical issues and future perspectives 
of EUS-guided ablation therapy will be discussed.

\section{INDICATION OF EUS-GUIDED ABLATION THERAPY}

\section{Surgical candidacy}

To achieve maximum therapeutic benefit and avoid unnecessary treatment, the indication for EUS-guided ablation therapy should be refined..$^{20}$ For pancreatic cystic neoplasm, the indication should be determined on the basis of the cyst type, morphology, and natural course. In patients with pancreatic cysts that meet the criteria for resection, surgical candidacy based on patients' underlying comorbidities should be assessed. For those that are deemed poor operative candidates, consideration for EUS-guided ablation therapy should be given. In addition, patients who are reluctant to undergo surgical resection and desire a less invasive treatment option should also be considered for EUS-guided ablation therapy.

\section{Cystic neoplasm}

In terms of cyst morphology, unilocular cysts with smaller sizes may attain the best outcome for the EUS-guided therapy. The accurate diagnosis of the type of pancreatic cyst (hence prognosticating its malignant potential) can be challenging for a significant portion of patients even after an exhaustive batteries of tests, including magnetic resonance imaging, EUS, and cyst fluid analysis. ${ }^{12,21-25}$ Therefore, the development of reliable biomarkers and new endoscopic technologies to enhance the accuracy of diagnosis and prognosticating malignant transformation potential for cystic neoplasm is still required. Regarding cyst type, the malignant potential of mucinous cysts is higher than that of serous cystic tumors. As mucinous cystic tumors often present as unilocular lesions, mucinous neoplasms that gradually increase in size are the ideal target for EUS-guided ablation therapy. A challenge in EUS-guided ablation of mucinous neoplasms, however, is that the reported treatment efficacy rates of EUS-guided ablation with ethanol injection were less robust than those for non-mucinous cystic neoplasms. ${ }^{9,12,13}$ Another issue with targeting mucinous neoplasms with EUS-guided treatment is their relatively lower prevalence among all pancreatic cystic neoplasms.

The most common pancreatic mucinous cystic neoplasm is the intraductal papillary mucinous neoplasm (IPMN), which communicates to the pancreatic duct. Though yet to be substantiated, the theoretical increased risk of posttreatment pancreatitis and injury to the pancreatic duct after ablation should demand careful consideration of the usefulness of the treatment for IPMNs. ${ }^{6}$ Although prophylactic pancreatic stent insertion before the procedure can be considered to reduce the risk of procedure-related pancreatitis, no reported data supporting its efficacy exist to date. Furthermore, a recent study by Park and colleagues on EUS-guided delivery of ethanol or radiofrequency ablation in a porcine model reported a potential risk of pancreatic ductal injury. ${ }^{26}$ Therefore, IPMN may be inappropriate for EUS-guided ablation therapy considering its low response rate and high risk of complications (Fig. 1).

\section{Solid neoplasm}

Regarding pancreatic solid neoplasms, small functioning pancreatic neuroendocrine tumors (PNETs) of $<2 \mathrm{~cm}$ are the most appropriate indication for EUS-guided ablation therapy. Functioning PNETs have hormone-related symptoms and

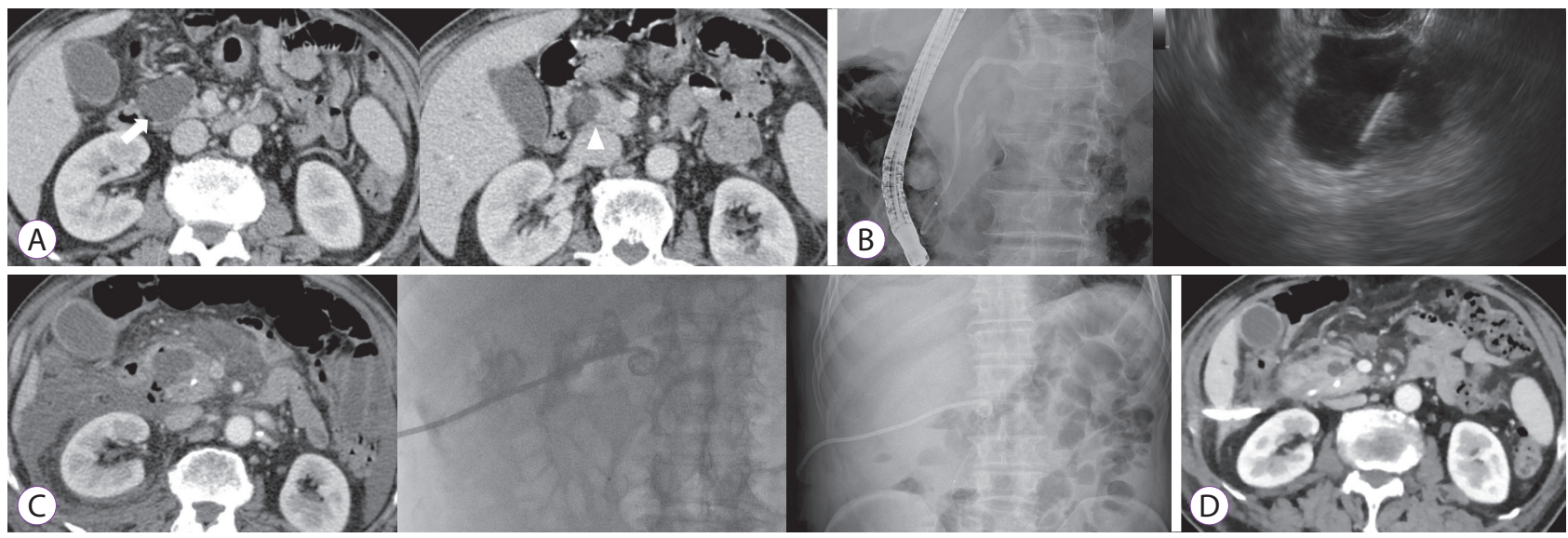

Fig. 1. A 77-year-old man with a pancreatic head cyst. (A) Computed tomography (CT) scan showing a 38-mm cystic lesion in the pancreatic head (white arrow), suspected as having communication with the pancreatic duct (white arrowhead). (B) Endoscopic retrograde pancreatic drainage with a 9-cm, 5-F, single pigtail plastic stent was performed to prevent procedure-related pancreatitis, and then endoscopic ultrasonography-guided ablation therapy was performed with $99 \%$ ethanol. (C) After the procedure, complicated fluid collection with pancreatitis-associated phlegmon and ascites along the right paracolic gutter was observed, and a percutaneous drainage tube was inserted. (D) CT scan showing improvement of the complicated fluid collection. 
malignant potential. ${ }^{19}$ As functioning NETs are usually small and sometimes manifest as multiple lesions, ${ }^{27}$ EUS-guided ablation therapy can be considered as a complementary tool to surgical resection. Evaluation of treatment response after ablation therapy of functioning PNETs can be performed by assessing the improvement of hormone-related symptom and/or with serial hormonal assays. ${ }^{15,16,19}$ EUS-guided ablation therapy for small nonfunctioning PNETs and solid pseudopapillary neoplasms is also under investigation. However, because severe complications, including severe acute pancreatitis, can occur, indiscriminate treatment should be avoided. ${ }^{18}$ Another concern of ablation therapy for these pancreatic solid neoplasms is local recurrence and inability to detect or treat metastatic lesions. As pancreatic solid neoplasms are rare and the natural history of these tumors is protean, selection of the patients must be based on strict criteria. Like cystic neoplasms of the pancreas, pancreatic solid tumors with main pancreatic duct involvement may be an inappropriate target because of the higher risk of pancreatic duct injury during ablation therapy. Moreover, because the involvement of the main pancreatic duct in these pancreatic neoplasms may indicate an aggressive clinical course, ${ }^{28,29}$ local ablative therapy may not be appropriate in these clinical circumstances.

\section{STANDARDIZATION OF THE TREATMENT PROTOCOL}

\section{Quantity, concentration, and method of delivery of the ablating agent}

In EUS-guided ablation therapy, ethanol has been the most commonly used ablative agents. However, no consensus has been reached on the treatment protocol specifying the concentration and amount of ethanol, method of delivery (lavage vs. instillation), duration and number of lavage, or the number of sessions necessary to optimize the efficacy and safety of the procedure. ${ }^{30}$ The ideal concentration of ethanol has not been clearly determined in EUS-guided ethanol ablation therapy. A previous animal study by Matthes and colleagues demonstrated that EUS-guided injection of ethanol in the pig pancreas resulted in a localized concentration-dependent tissue necrosis. ${ }^{31}$ The concentration of injected ethanol, final concentration of ethanol inside the cyst, and complete resolution rate of the cyst in previous clinical studies are described in Table 1. 912,30,32-34 $^{-3}$ Most studies did not measure final concentrations of ethanol inside the cyst, which can vary depending on the amount of ethanol injected and the volume of residual cystic fluid prior to delivery of ethanol. In a recent single-center, prospective study, cyst ablation with $80 \%$ ethanol showed a very low complete resolution of pancreatic cyst (2/23), and the mean final concentration of ethanol achieved in the treated cysts was only $50 \%$ (range, $0 \%-79 \%$ ). It seems that the higher the concentration of the injected ethanol, the better the treatment efficacy. At present, whether higher concentrations are also associated with increased risk of complications such as post-ablation pancreatitis or pancreatic duct injury is uncertain.

Ideally, the amount of ablating agent (i.e., ethanol) injected to the lesion should be of the same volume as the cystic fluid aspirated prior to its delivery. ${ }^{13}$ In the ablation of solid tumors, the amount of ethanol that needs to be delivered should be calculated on the basis of the volume of the tumor by using cross-sectional imaging. Lastly, one must consider the known fine needle aspiration needle dead space volume that occupies the needle channel $(1.5-2 \mathrm{~mL}){ }^{18}$

As previously mentioned, communication of the lesion to the pancreatic duct carries a potential risk of adverse outcome. Recently, a new technique has been introduced to delineate lesions communicating with the main pancreatic duct from those that do not. ${ }^{9}$ After EUS-guided cyst aspiration, a radiocontrast medium was injected through the needle to confirm no leakage to the pancreatic parenchyma or communication with the pancreatic duct. Although this can aid in selecting lesions with a higher risk of adverse outcome, it may also diminish treatment efficacy, as the injected radiocontrast may reduce or even prevent full exposure of the epithelial lining of the pancreatic cyst to ethanol. ${ }^{35}$

Table 1. Treatment Outcome of Endoscopic Ultrasonography-Guided Ethanol Ablation Therapy for Pancreatic Cystic Neoplasm according to Ethanol Concentration

\begin{tabular}{lcccc}
\hline Study & Patients $(\boldsymbol{n})$ & $\begin{array}{c}\text { Concentration of injected } \\
\text { ethanol }\end{array}$ & $\begin{array}{c}\text { Final concentration of etha- } \\
\text { nol inside the cyst }\end{array}$ & $\begin{array}{c}\text { Complete resolution rate of } \\
\text { the cyst }\end{array}$ \\
\hline Park et al. $(2016)^{12}$ & 91 & $99 \%$ & $98 \%$ & $41(45 \%)$ \\
Gómez et al. $(2016)^{9}$ & 23 & $80 \%$ & Mean $50 \%$ (range, 0\%-79\%) & $2(9 \%)$ \\
Caillol et al. $(2012)^{32}$ & 13 & $99 \%$ & Not mentioned & $11(85 \%)$ \\
DiMaio et al. $(2011)^{33}$ & 13 & $80 \%$ & Not mentioned & $5(38 \%)$ \\
DeWitt et al. $(2009)^{34}$ & 36 & $80 \%$ & Not mentioned & $12(33 \%)$ \\
Gan et al. $(2005)^{30}$ & 23 & Mean $46 \%$ (range, $5 \%-80 \%)$ & Not mentioned & $8(35 \%)$ \\
\hline
\end{tabular}


Regarding EUS-guided radiofrequency ablation, previous clinical trials showed acceptable feasibility, safety, and efficacy in targeting nonfunctioning PNETs, insulinomas, and pancreatic mucinous cystic tumors. ${ }^{36-38}$ Further clinical studies are required to standardize the designs of catheter-based electrodes, the ablation energy required, the ablation time, and the number of EUS-guided radiofrequency ablation therapy sessions.

The ideal ablative agent that provides the optimal treatment effect is yet to be clearly elucidated. Most of the studies to date on EUS-guided ablation therapy are relatively small, observational studies without proper control groups or head-to-head comparison with other treatment methods. The addition of paclitaxel after ethanol injection, for example, has demonstrated increased therapeutic effect in some studies. ${ }^{10,11,14} \mathrm{Re}$ cently, ethanol-free EUS-guided cyst chemoablation with an admixture of paclitaxel and gemcitabine has demonstrated a significant reduction in posttreatment adverse events, with similar efficacy with ethanol lavage followed by paclitaxel injection. ${ }^{39}$ Regarding pancreatic solid neoplasm, ethanol abla- tion therapy combined with lipiodol may be useful, especially in hypervascular tumors, including neuroendocrine tumors. ${ }^{16}$ Larger prospective studies to compare the efficacy and safety of each treatment modality, including injection of ablative or chemotherapeutic agents, and radiofrequency ablation therapy, will be required to clarify this issue. ${ }^{5,40}$

\section{Safety-related issue}

The short-term adverse events of EUS-guided ablation therapy include abdominal pain, acute pancreatitis, vascular injury, and infection. ${ }^{41}$ Most of these adverse events are mild and self-limited, but the development of severe complications require hospital admission with close observation and necessary intervention. Severe pancreatitis can develop owing to leakage of the proinflammatory ablative agents into the surrounding pancreatic parenchyma or direct injury of the pancreatic duct. ${ }^{18}$ Exophytic lesions of the pancreas (either cystic or solid) may be inappropriate for EUS-guided ablation therapy because of the higher risk of leakage (Fig. 2). ${ }^{6}$ Radiofrequency
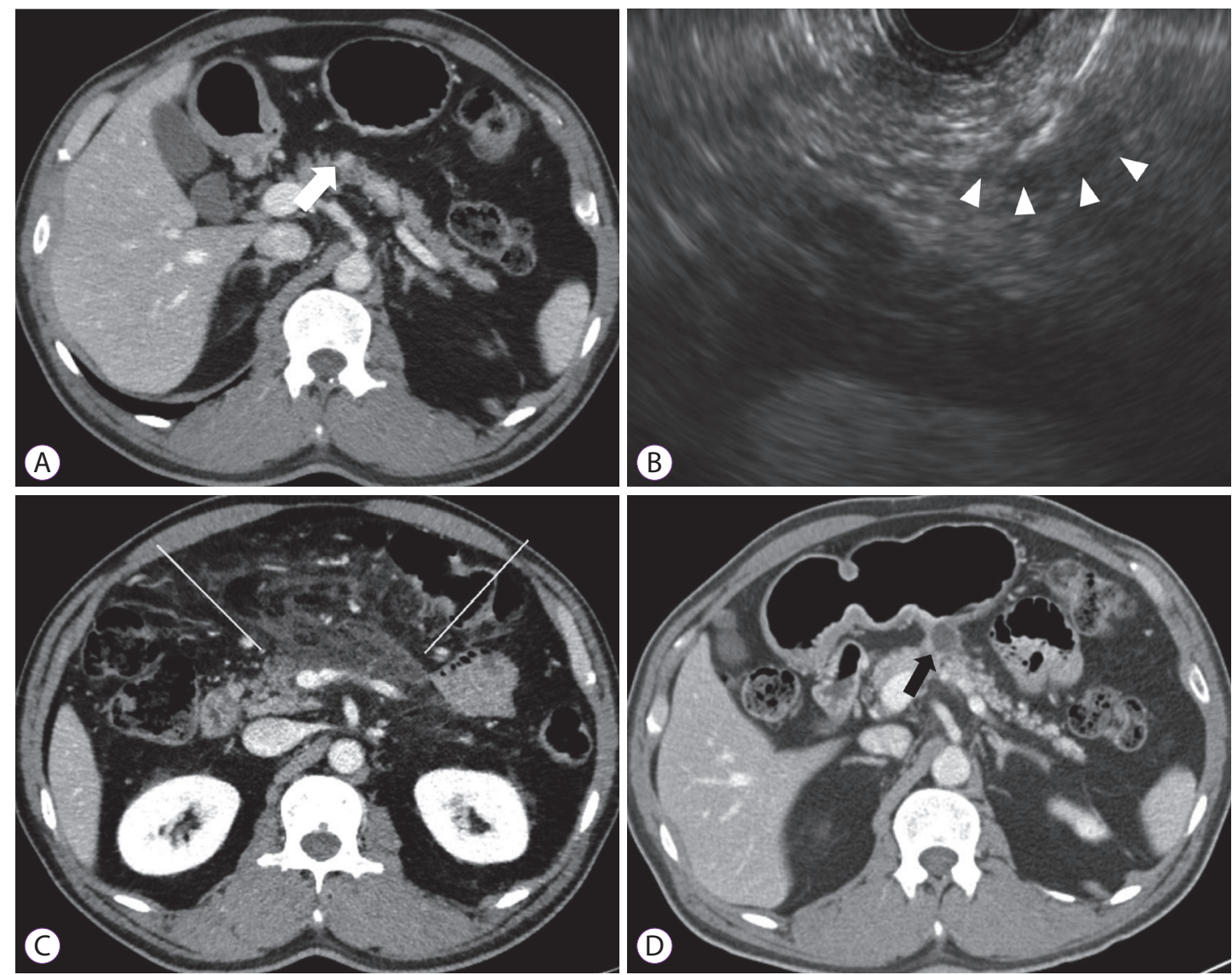

Fig. 2. A 58-year-old man with a pancreatic neuroendocrine tumor (PNET). (A) Computed tomography (CT) scan showing a 13-mm enhancing nodular lesion with an exophytic feature (white arrow) in the pancreatic body without dilation of the main pancreatic duct. (B) Endoscopic ultrasonography-guided ablation therapy for PNET (white arrowhead) was performed, and $0.6 \mathrm{~mL}$ of $99 \%$ ethanol was injected in the lesion. (C) A long perfusion decrement of the pancreatic body (lines) on the CT scan obtained 2 days after the procedure. (D) Follow-up CT scan showing improvement of the walled-off necrosis with 2-cm loculated fluid collection (black arrow) at the previous ablation site. 
ablation demonstrates the ability to induce thermal injury on the target lesion. Subsequently, a potential risk of thermal damage to adjacent structures can result in necrotizing pancreatitis with peritonitis, burning of the gastric wall, bleeding, portal vein thrombosis, or even fistula. ${ }^{42,43}$ Consequently, in EUS-guided radiofrequency ablation, lesions must be ablated with sufficient safety margin.

Long-term data on EUS-guided cyst ablation have been reported by two centers from Korea and one from the United States. ${ }^{9,12,13}$ Choi and colleagues reported a $98 \%$ remission rate at 6-year follow-up among 114 patients with pancreatic cysts in whom complete resolution was achieved after EUS-guided cyst ablation..$^{13}$ In the other study from Korea, Park and colleagues reported no further increase in size or malignant transformation in 7 patients with persistent pancreatic cysts after EUS-assisted ablation during the median follow-up period of 46 months. ${ }^{12}$ It is interesting that Gómez and colleagues from the United States reported non-response to the ablation treatment in a patient with branch duct IPMNs who eventually developed pancreatic adenocarcinoma 41 months later. ${ }^{9}$ As the numerical assessment of the cancer prevention rate by EUS-assisted ablation therapy is not possible at present, the need for surveillance, including frequency, duration, and modality, is obscure and thus must be clarified. The long-term treatment outcome of EUS-guided ablation therapy in terms of recurrence, malignant transformation, or metastasis should be validated in multicenter controlled studies.

\section{Evaluation of treatment outcome and follow-up}

After ablation therapy, though often difficult, the effectiveness of the treatment and follow-up interval should be assessed, as reliable markers predicting treatment outcome are lacking. In terms of pancreatic cyst ablation, studies have relied on cyst size determined using cross-sectional imaging, including computed tomography or magnetic resonance imaging. Unfortunately, owing to the innate limitation in their ability to detect miniscule residual lesions, dependence on follow-up imaging alone may not be adequate to reliably report the eradication of all cystic contents and wall epithelium. ${ }^{35}$ This is especially true in the follow-up of solid neoplasms where residual clusters of neoplastic lesion cannot be detected if the residual lesion is too small. Surrogate biomarkers that adequately forecast the underlying etiology, potential malignant risk, and probability of cancer prevention by ablation therapy should be developed. ${ }^{8}$ Contrast-enhanced harmonic EUS, needle-based confocal laser endomicroscopy probes, and microforceps passed through an EUS needle may be helpful to determine the treatment outcome of EUS-guided ablation therapy. ${ }^{18,44-46} \mathrm{~A}$ combination of serological and radiographic and/or endoscopic imaging would most likely provide the most cogent solution to the dilemma.

\section{CONCLUSIONS}

EUS-guided ethanol ablation therapy seems to be a promising option for patients with pancreatic neoplasms, including mucinous cystic neoplasm and small functioning PNETs. EUS-guided ablation therapy could be used to complement radical resection of pancreatic neoplasms. However, safety-related issues should be considered, and a consensus and guideline for EUS-guided ethanol ablation therapy should be established. Development of dedicated devices and ablative agents for EUS-guided ablation therapy, and reliable biomarkers for evaluating the treatment responsiveness is also important.

\section{Conflicts of Interest}

The authors have no financial conflicts of interest.

\section{REFERENCES}

1. de Jong K, Nio CY, Hermans JJ, et al. High prevalence of pancreatic cysts detected by screening magnetic resonance imaging examinations. Clin Gastroenterol Hepatol 2010;8:806-811.

2. Laffan TA, Horton KM, Klein AP, et al. Prevalence of unsuspected pancreatic cysts on MDCT. AJR Am J Roentgenol 2008;191:802-807.

3. Dasari A, Shen C, Halperin D, et al. Trends in the incidence, prevalence, and survival outcomes in patients with neuroendocrine tumors in the United States. JAMA Oncol 2017;3:1335-1342.

4. Haynes AB, Deshpande V, Ingkakul T, et al. Implications of incidentally discovered, nonfunctioning pancreatic endocrine tumors: short-term and long-term patient outcomes. Arch Surg 2011;146:534-538.

5. Han J, Chang KJ. Endoscopic ultrasound-guided direct intervention for solid pancreatic tumors. Clin Endosc 2017;50:126-137.

6. Lee SH. [Endoscopic treatment for pancreatic cystic lesions]. Korean J Gastroenterol 2018;71:10-17.

7. Choi JH, Lee SH, Choi YH, et al. Clinical outcomes of endoscopic ultrasound-guided ethanol ablation for pancreatic cystic lesions compared with the natural course: a propensity score matching analysis. Therap Adv Gastroenterol 2018;11:1756284818759929.

8. Lee LS, Andersen DK, Ashida R, et al. EUS and related technologies for the diagnosis and treatment of pancreatic disease: research gaps and opportunities-summary of a national institute of diabetes and digestive and kidney diseases workshop. Gastrointest Endosc 2017;86:768-778.

9. Gómez V, Takahashi N, Levy MJ, et al. EUS-guided ethanol lavage does not reliably ablate pancreatic cystic neoplasms (with video). Gastrointest Endosc 2016;83:914-920.

10. Oh HC, Seo DW, Song TJ, et al. Endoscopic ultrasonography-guided ethanol lavage with paclitaxel injection treats patients with pancreatic cysts. Gastroenterology 2011;140:172-179.

11. Oh HC, Seo DW, Lee TY, et al. New treatment for cystic tumors of the pancreas: EUS-guided ethanol lavage with paclitaxel injection. Gastrointest Endosc 2008;67:636-642.

12. Park JK, Song BJ, Ryu JK, et al. Clinical outcomes of endoscopic ultrasonography-guided pancreatic cyst ablation. Pancreas 2016;45:889-894.

13. Choi JH, Seo DW, Song TJ, et al. Long-term outcomes after endoscopic ultrasound-guided ablation of pancreatic cysts. Endoscopy 2017;49:866- 
873.

14. DeWitt JM, Al-Haddad M, Sherman S, et al. Alterations in cyst fluid genetics following endoscopic ultrasound-guided pancreatic cyst ablation with ethanol and paclitaxel. Endoscopy 2014;46:457-464.

15. Levy MJ, Thompson GB, Topazian MD, Callstrom MR, Grant CS, Vella A. US-guided ethanol ablation of insulinomas: a new treatment option. Gastrointest Endosc 2012;75:200-206.

16. Park DH, Choi JH, Oh D, et al. Endoscopic ultrasonography-guided ethanol ablation for small pancreatic neuroendocrine tumors: results of a pilot study. Clin Endosc 2015;48:158-164.

17. Yang D, Inabnet WB 3rd, Sarpel U, DiMaio CJ. EUS-guided ethanol ablation of symptomatic pancreatic insulinomas. Gastrointest Endosc 2015;82:1127.

18. Paik WH, Seo DW, Dhir V, Wang HP. Safety and efficacy of EUS-guided ethanol ablation for treating small solid pancreatic neoplasm. Medicine (Baltimore) 2016;95:e2538.

19. Paik WH, Seo DW. Echoendoscopic ablative therapy for solid pancreatic tumors. J Dig Dis 2017;18:135-142.

20. Cho MK, Choi JH, Seo DW. Endoscopic ultrasound-guided ablation therapy for pancreatic cysts. Endosc Ultrasound 2015;4:293-298.

21. Lee LS, Wu BU, Banks PA, et al. Utility of commercial DNA analysis in detecting malignancy within pancreatic cysts. JOP 2013;15:182-188.

22. Correa-Gallego C, Ferrone CR, Thayer SP, Wargo JA, Warshaw AL, Fernández-Del Castillo C. Incidental pancreatic cysts: do we really know what we are watching? Pancreatology 2010;10:144-150.

23. Chiang AL, Lee LS. Clinical approach to incidental pancreatic cysts. World J Gastroenterol 2016;22:1236-1245.

24. Jang DK, Song BJ, Ryu JK, et al. Preoperative diagnosis of pancreatic cystic lesions: the accuracy of endoscopic ultrasound and cross-sectional imaging. Pancreas 2015;44:1329-1333.

25. Del Chiaro M, Segersvärd R, Pozzi Mucelli R, et al. Comparison of preoperative conference-based diagnosis with histology of cystic tumors of the pancreas. Ann Surg Oncol 2014;21:1539-1544.

26. Park JS, Seo DW, Song TJ, et al. Endoscopic ultrasound-guided ablation of branch-duct intraductal papillary mucinous neoplasms: feasibility and safety tests using porcine gallbladders. Dig Endosc 2016;28:599-606.

27. Kim MK. Endoscopic ultrasound in gastroenteropancreatic neuroendocrine tumors. Gut Liver 2012;6:405-410.

28. Nanno Y, Matsumoto I, Zen Y, et al. Pancreatic duct involvement in well-differentiated neuroendocrine tumors is an independent poor prognostic factor. Ann Surg Oncol 2017;24:1127-1133.

29. Canellas R, Burk KS, Parakh A, Sahani DV. Prediction of pancreatic neuroendocrine tumor grade based on CT features and texture analysis. AJR Am J Roentgenol 2018;210:341-346.

30. Gan SI, Thompson CC, Lauwers GY, Bounds BC, Brugge WR. Ethanol lavage of pancreatic cystic lesions: initial pilot study. Gastrointest Endosc 2005;61:746-752.

31. Matthes K, Mino-Kenudson M, Sahani DV, Holalkere N, Brugge WR.
Concentration-dependent ablation of pancreatic tissue by EUS-guided ethanol injection. Gastrointest Endosc 2007;65:272-277.

32. Caillol F, Poincloux L, Bories E, et al. Ethanol lavage of 14 mucinous cysts of the pancreas: a retrospective study in two tertiary centers. Endosc Ultrasound 2012;1:48-52.

33. DiMaio CJ, DeWitt JM, Brugge WR. Ablation of pancreatic cystic lesions: the use of multiple endoscopic ultrasound-guided ethanol lavage sessions. Pancreas 2011;40:664-668.

34. DeWitt J, McGreevy K, Schmidt CM, Brugge WR. EUS-guided ethanol versus saline solution lavage for pancreatic cysts: a randomized, double-blind study. Gastrointest Endosc 2009;70:710-723.

35. Vazquez-Sequeiros E, Maluf-Filho F. Endosonography-guided ablation of pancreatic cystic tumors: is it justified? Gastrointest Endosc 2016;83:921-923.

36. Armellini E, Crinò SF, Ballarè M, Occhipinti P. Endoscopic ultrasound-guided radiofrequency ablation of a pancreatic neuroendocrine tumor. Endoscopy 2015;47 Suppl 1 UCTN:E600-E601.

37. Pai M, Habib N, Senturk H, et al. Endoscopic ultrasound guided radiofrequency ablation, for pancreatic cystic neoplasms and neuroendocrine tumors. World J Gastrointest Surg 2015;7:52-59.

38. Lakhtakia S, Ramchandani M, Galasso D, et al. EUS-guided radiofrequency ablation for management of pancreatic insulinoma by using a novel needle electrode (with videos). Gastrointest Endosc 2016;83:234239.

39. Moyer MT, Sharzehi S, Mathew A, et al. The safety and efficacy of an alcohol-free pancreatic cyst ablation protocol. Gastroenterology 2017;153:1295-1303.

40. Lakhtakia S, Seo DW. Endoscopic ultrasonography-guided tumor ablation. Dig Endosc 2017;29:486-494.

41. Oh HC, Seo DW, Kim SC. Portal vein thrombosis after EUS-guided pancreatic cyst ablation. Dig Dis Sci 2012;57:1965-1967.

42. Silviu UB, Daniel P, Claudiu M, et al. Endoscopic ultrasound-guided radiofrequency ablation of the pancreas: an experimental study with pathological correlation. Endosc Ultrasound 2015;4:330-335.

43. Girelli R, Frigerio I, Salvia R, Barbi E, Tinazzi Martini P, Bassi C. Feasibility and safety of radiofrequency ablation for locally advanced pancreatic cancer. Br J Surg 2010;97:220-225.

44. Nakai Y, Iwashita T, Park DH, Samarasena JB, Lee JG, Chang KJ. Diagnosis of pancreatic cysts: EUS-guided, through-the-needle confocal laser-induced endomicroscopy and cystoscopy trial: DETECT study. Gastrointest Endosc 2015;81:1204-1214.

45. Attili F, Pagliari D, Rimbaș M, et al. Endoscopic ultrasound-guided histological diagnosis of a mucinous non-neoplastic pancreatic cyst using a specially designed through-the-needle microforceps. Endoscopy 2016;48 Suppl 1:E188-E189.

46. Karia K, Waxman I, Konda VJ, et al. Needle-based confocal endomicroscopy for pancreatic cysts: the current agreement in interpretation. Gastrointest Endosc 2016;83:924-927. 